\title{
Avaliação do uso de Objeto de Aprendizagem no ensino da Taxonomia North American Nursing Diagnosis Association (Nanda)
}

\author{
Assessment of Learning Objects use in the teaching of Taxonomy North \\ American Nursing Diagnosis Association (Nanda)
}

\author{
${ }^{1}$ Anicésia Cecília Gotardi Ludovino \\ ${ }^{2}$ Leonardo Feriato Moreira \\ ${ }^{1}$ Maria José Bistafa Pereira \\ ${ }^{3}$ Pablo Rodrigo Sanches \\ ${ }^{1}$ Silvia Sidnéia da Silva \\ ${ }^{1}$ Edilson Carlos Caritá
}

cecilialudo@hotmail.com

\section{RESUMO}

O estudo teve como objetivo avaliar o uso de um Objeto de Aprendizagem (OA) como apoio ao ensino da Taxonomia North American Nursing Diagnosis Association (NANDA) na realização do diagnóstico de enfermagem. Trata-se de estudo exploratório-descritivo, com abordagem quantiqualitativa, realizado com alunos de graduação em Enfermagem de uma instituição de ensino superior privada do interior mineiro. Na primeira etapa os alunos realizaram uma avaliação (pré-OA) com dois estudos de caso; na segunda, utilizaram o OA desenvolvido para esse estudo durante quinze dias e na terceira etapa fizeram, após o período de quinze dias, outra avaliação (pós-OA) utilizando os mesmos estudos de caso. Os resultados demonstraram que antes de utilizar o OA a média de acertos (estudo de caso 1) foi de 3,79 \pm 2,14 e após o uso do OA atingiram média de 5,21 $\pm 2,74$. Também foi realizado o teste t de Student, considerando-se um p-value de 0,05, o p-value observado foi de 0,0030 , indicando que as médias das duas avaliações diferem estatisticamente, o que também ocorreu com a segunda avaliação (estudo de caso 2), sendo a média antes do uso do OA de 1,60 $\pm 1,19$ e, após o uso do OA passou para $2,79 \pm 1,44$, com o resultado do teste $t$ de Student com p-value observado de 0,0 . O estudo evidenciou que o OA avaliado poderá ser utilizado como recurso didático-pedagógico no ensino da assistência de enfermagem, pois a maioria dos alunos teve melhor desempenho nas avaliações após sua utilização.

Palavras-chave: Objeto de Aprendizagem. Tecnologia da Informação e da Comunicação. Taxonomia NANDA. Aplicativo.

\section{ABSTRACT}

The objective of this study was to evaluate the use of a Learning Object (LO) to support the teaching of the North American Nursing Diagnosis Association (NANDA) in the nursing diagnosis. This is an exploratory-descriptive study, with quali-quantitative approach, carried out with nursing undergraduate students from a private higher education institution in the interior of Minas Gerais. In the first stage the students performed an evaluation (pre-LO) with two case studies; in the second, they used the LO developed for that study during fifteen days and in the third stage did, after the fifteen days, another evaluation (post-LO) using the same case studies. The results showed that before using the $L O$, the mean number of correct answers (case study 1) was $3.79 \pm 2.14$ and after $L O$, mean values were $5.21 \pm 2.74$. A Student $t$-test was also performed, considering a p-value of 0.05, p-value observed was 0.0030, indicating that the means of the two evaluations differed statistically, which also occurred with the second evaluation (case study 2). The mean before use of LO was $1.60 \pm$ 1.19 and, after $L O$, it was $2.79 \pm 1.44$, with the result of Student $t$-test with $p$-value observed at 0.0. The study evidenced that the evaluated $L O$ can be used as a didactic-pedagogical resource in the nursing care teaching, since most of the students had better performance in the evaluations after the use of the LO.

Keywords: Learning Object. Information and Communication Technology. NANDA Taxonomy. App.

\footnotetext{
Programa de Mestrado Profissional em Saúde e Educação, Universidade de Ribeirão Preto (UNAERP).

Curso de Engenharia de Computação, Universidade de Ribeirão Preto (UNAERP).

Curso de Engenharia de Software, Universidade de Ribeirão Preto (UNAERP).
} 


\section{INTRODUÇÃO}

Vivenciamos a transição da Era da Informação para a Era do Conhecimento, onde a tecnologia possibilita a expressão de novas atividades, novos formatos de interação social, ampliação dos locais, formatos e estrutura do processo ensino-aprendizagem, ampliando a percepção da sala de aula e das interações aluno-professor e aluno-aluno, adequando-a à necessidade do mercado, tendo mudanças da educação tradicional para uma educação ativa denominada metodologias ativas de aprendizagem (SABOIA; VARGAS; VIVA, 2013).

As Instituições de Ensino Superior (IES) têm sido motivadas a refletir e implementar mudanças em suas matrizes curriculares, para que sejam adotadas alternativas inovadoras de ensino que priorizem as metodologias ativas de aprendizagem, possibilitando um aprendizado facilitador na direção da aquisição de habilidades, competências e atitudes baseadas em resultados. Entende-se que os cenários de aprendizagem devem incorporar e interligar métodos didático-pedagógicos de áreas de práticas e vivências; de utilização de tecnologias e habilidades cognitivas e psicomotoras; de valorização dos preceitos morais e éticos, orientadores de condutas individuais e coletivas; bem como de organização do processo de trabalho (MILLÃO et al., 2017).

Os estudantes e profissionais da saúde têm buscado diferentes formas de se adequar às atividades diárias, demonstrando diversas competências, entre elas, o conhecimento acerca das novas tecnologias. As TIC disponibilizam inúmeras estratégias para uma formação ampla do profissional, destacando-se, a informática em saúde e a Internet, proporcionando novos espaços para o desenvolvimento de um processo ensino-aprendizagem que supere o tradicional e quebre paradigmas de tempo, espaço e avaliação da aprendizagem (WESTIN et al., 2016).

Ainda segundo Westin et al. (2016), as TIC são estratégias para diferenciar os processos ensino-aprendizagem tradicionais, muitas vezes obsoletos, por novas formas, mais colaborativas e ativas de aprendizagem.

Em um curso de graduação em enfermagem, um dos conteúdos de estudo contemplados é a Sistematização de Assistência de Enfermagem (SAE), que consta do processo de enfermagem composto por um conjunto de etapas que envolvem: coleta de dados por meio da anamnese e exame físico, definição de diagnósticos de enfermagem tendo como referencial a Taxonomia North American Nursing Diagnosis Association (NANDA), o planejamento, a implementação das intervenções de enfermagem e a avaliação dos resultados, mediante uma abordagem voltada à solução de problemas e ao estabelecimento de metas para atingir melhores resultados, oferecendo uma assistência de enfermagem integral e individualizada a cada paciente com o objetivo de solucionar os problemas existentes para atingir a assistência de qualidade (PAGANIN et al., 2010).

As múltiplas responsabilidades e o dever do enfermeiro para realizar suas atribuições nos serviços de enfermagem associados à falta de preparo profissional para a utilização do diagnóstico de enfermagem que compõem a SAE dificultam a excelência no atendimento da enfermagem. No entanto, a ciência e a tecnologia avançam numa velocidade espantosa, tornando a vida melhor, e as TIC são um caminho para apoiar e facilitar o processo de enfermagem, especificamente, no diagnóstico de enfermagem utilizando a Taxonomia NANDA (DAL SASSO et al., 2013).

Na década de 1970, iniciou-se nos Estados Unidos da América (EUA) o movimento dos diagnósticos de enfermagem, tendo como finalidade, no princípio, a necessidade de explicitar para as seguradoras de saúde daquele país o que as enfermeiras realizavam na sua prática assistencial. Este movimento deu origem à NANDA que concebeu, então, a primeira taxonomia de diagnósticos de enfermagem, que se tornou a mais conhecida e utilizada mundialmente (BARROS; FAKIH; MICHEL, 2002).

A enfermagem, ao buscar a profissionalização e valorização dos profissionais no Brasil, teve a expressão “diagnóstico de enfermagem” introduzida por Wanda Aguiar Horta, na década de 60, que se constituiu em uma das etapas do processo de enfermagem proposto por esta autora (HORTA,1979). 
Segundo o Conselho Federal de Enfermagem (COFEn) (2009), por meio da Resolução COFEn ${ }^{\circ}$ 358/2009, a SAE organiza o trabalho profissional quanto ao método, pessoal e instrumentos, tornando possível a operacionalização do Processo de Enfermagem (PE), traduzindo-se em um instrumento metodológico que orienta o cuidado profissional de Enfermagem e a documentação da prática profissional, além de evidenciar a contribuição da Enfermagem na atenção à saúde da população, aumentando a visibilidade e o reconhecimento profissional por meio da operacionalização e documentação desse PE.

Conforme Dal Sasso et al. (2013), o PE destaca-se como uma tecnologia do cuidado que orienta a sequência do raciocínio lógico e melhora a qualidade desse cuidado por meio da sistematização da avaliação clínica, dos diagnósticos, das intervenções e dos resultados de enfermagem, constituindo-se em ferramenta que deve ser utilizada pelos enfermeiros, pois enfatiza o desencadeamento dos pensamentos e juízos desenvolvidos durante a realização dos cuidados, integra, organiza e garante a continuidade das informações da equipe de enfermagem, permitindo avaliar a sua eficácia e efetividade e, modificá-la de acordo com os resultados na recuperação do cliente. Serve também de fundamentação permanente para a educação, pesquisa e gerenciamento em enfermagem.

O processo de raciocínio diagnóstico é complexo, e envolve a interpretação das respostas em relação à saúde e aos problemas que com ela concorrem, podendo ter impressões subjetivas do diagnosticador, sustentadas em conhecimentos, experiências, crenças e teorias pessoais. No entanto, quando o raciocínio diagnóstico é conduzido de forma não-estruturada está propenso a mais riscos de dispersão ou erros de julgamento. A linguagem padronizada e universalmente aceita, como da NANDA, são classificações que fornecem uma estrutura para a organização do conteúdo concernente aos diagnósticos como fenômenos de interesse da disciplina de enfermagem, pois fornecem recursos para pensar os conhecimentos existentes e, se necessário, reorientá-los (CLEIRES et al., 2015).

Os Objetos de Aprendizagem (OA) podem ser empregados desde a educação básica até cursos superiores das diferentes área do saber, pois promovem aprendizagem colaborativa, cuja ferramenta pedagógica permite a construção do conhecimento contemplando a interação e a cooperação entre parceiros, com o objetivo de localizar conteúdos educacionais na Web para serem reutilizados em diferentes cursos e plataformas, possibilitando, assim, a redução de custos de produção de materiais educacionais; ainda podem tanto acelerar processos individuais de aprendizagem quanto possibilitar que os alunos ajudem-se mutuamente sob a mediação do professor na sala de aula (SAMPAIO; ALMEIDA, 2010; SALVADOR et al., 2017).

Tubelo et al. (2012) e Fonseca et al. (2012) afirmam que os OA são recursos didático-pedagógicos digitais com suporte multimídia e linguagem hipermídia que podem ser reutilizados e têm meios para aprendizagem interativa com animações e simulações.

Alvarez e Dal Sasso (2011) citam ainda que com a diversidade de recursos disponíveis na informática, os OA oferecem oportunidades no processo ensino-aprendizagem sendo um recurso digital que pode ser utilizado para o suporte ao ensino visando suprir as possíveis deficiências de aprendizagem, propiciando ao graduando a construção do percurso cognitivo para aplicabilidade no processo ensino-aprendizagem em saúde e enfermagem.

Considerando as dificuldades apresentadas pelos estudantes no aprendizado da SAE e uso da Taxonomia NANDA, entende-se que é contemporâneo e relevante avaliar o uso de OA na ensinagem da SAE, como ferramenta de apoio ao processo ensino-aprendizagem.

O objetivo do estudo foi avaliar o uso de um Objeto de Aprendizagem (OA) como apoio ao ensino da Taxonomia North American Nursing Diagnosis Association (NANDA) na realização do diagnóstico de enfermagem. 


\section{MATERIAL E MÉTODOS}

Trata-se de estudo exploratório-descritivo, de abordagem qualiquantitativa.

A proposta do estudo foi aprovada pelo Comitê de Ética e Pesquisa sob o CAAE n ${ }^{0}$ 77966317.7.0000.5498 e parecer n ${ }^{\circ} 2.394 .834$ seguindo-se os preceitos éticos, estabelecidos pela Resolução n ${ }^{\circ}$ 466/2012, do Conselho Nacional de Saúde.

A população do estudo constituiu-se dos alunos do curso de graduação em Enfermagem de uma Instituição de Ensino Superior (IES) privada do interior mineiro, que estavam cursando ou já haviam cursado a disciplina de Sistematização da Assistência de Enfermagem, matriculados no primeiro semestre de 2018, totalizando 42 alunos.

Todos os discentes do curso de graduação em Enfermagem matriculados do quarto ao décimo período foram convidados a participar deste estudo, aceitaram e assinaram o Termo de Consentimento Livre e Esclarecido (TCLE).

A pesquisadora se encontrou pessoalmente com os participantes da pesquisa nas dependências da IES onde se realizou o estudo, em sala autorizada pela Instituição, para a realização da coleta de dados. Os participantes receberam e responderam ao instrumento de coleta de dados, ficando a pesquisadora disponível para esclarecer eventuais dúvidas.

Na primeira etapa os participantes do estudo realizaram a avaliação denominada pré-OA sobre estudos de caso validados, em que os alunos realizaram o diagnóstico de enfermagem segundo a Taxonomia NANDA e, na sequência foram convidados a utilizar o OA denominado iNanda para apoiar o processo ensino-aprendizagem da SAE, por um período de quinze dias.

Após os quinze dias de uso do OA realizaram novamente uma avaliação, denominada pós-OA, sobre os mesmos estudos de caso, apresentados no pré-OA. Os resultados das avaliações foram analisados estatisticamente para verificar se o uso do OA contribuiu para o processo ensino-aprendizagem da Taxonomia NANDA e, na última etapa do estudo, os participantes fizeram uma avaliação qualiquantitativa sobre suas percepções em relação ao uso do OA iNanda no seu processo ensino-aprendizagem.

O OA iNanda é um app e foi desenvolvido por meio do ambiente de desenvolvimento Android Studio versão 3.0.1 com o Software Development Kit (SDK) do Android, pode ser instalado em qualquer dispositivo que possua o sistema operacional Android na versão 4.4 (Android KitKat ${ }^{\circledR}$ ) ou superior. O app recebe os dados contidos no banco de dados por meio de um web service, quando conectado à Internet.

O banco de dados foi elaborado utilizando o Real-Time Database versão 13.0 da plataforma de web service Firebase versão 13.0 da empresa Google. O Real-Time Database é um serviço de web services oferecido na plataforma Firebase, cuja principal característica é a atualização em tempo real dos dados, já cadastrados na base de dados, em todos os dispositivos que estiverem conectados a ele, por meio da Internet. Além disso, possibilita que esses dados fiquem armazenados nos dispositivos, permitindo que o app ainda seja totalmente funcional mesmo que não esteja conectado à Internet.

O web service envia os pedidos de requisição que a aplicação faz ao banco de dados e este retorna ao web service os respectivos dados solicitados. O web service retorna os dados no formato JavaScript Object Notation (JSON), que é um dos formatos mais utilizados contemporaneamente para troca de informações entre sistemas de informação, sendo que a aplicação consegue interpretar e aplicar esses dados aos seus processos.

O app está disponível para download e instalação no serviço de distribuição digital de aplicativos Google Play. O OA contempla informações sobre o diagnóstico de enfermagem da Taxonomia NANDA, permite realizar 
consultas aos diagnósticos de enfermagem cadastrados utilizando como palavras-chave o código, o título ou as características definidoras do diagnóstico.

O app possui duas interfaces principais, a primeira é a tela inicial, onde são listados o código de identificação, o número da classe e do domínio ao qual o diagnóstico pertence, bem como o título dos diagnósticos da Taxonomia NANDA, cadastrados na base de dados. O estudante de enfermagem que usar o app conseguirá acessar qualquer diagnóstico cadastrado.

Para facilitar a consulta aos diagnósticos de enfermagem na tela há um botão com o ícone de uma lupa, e ao digitar o nome ou o código do diagnóstico é possível identificá-lo sem a necessidade de trocar de tela (Figura 1).

Na segunda tela está disponível o acesso a uma explicação detalhada do diagnóstico de enfermagem selecionado na lista da tela inicial (Figura 2). Há um botão com um ícone de uma prancheta e ao clicá-lo ocorre o redirecionamento para outra tela, onde o estudante pode pesquisar o diagnóstico, informando no mínimo, três características definidoras (Figura 3).

Na Figura 4 é apresentado o resultado de uma consulta realizada por meio das características definidoras.

Figura 1 - Tela inicial

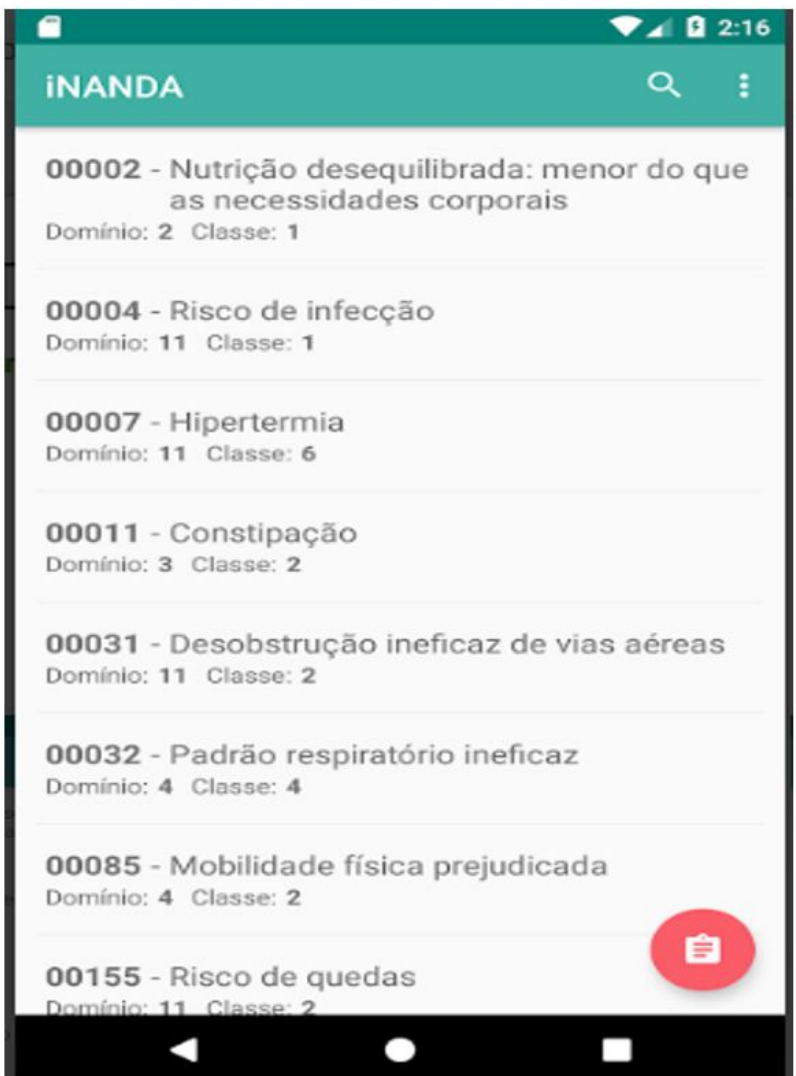

Fonte: Autoria Própria

\section{Figura 2 - Definição do diagnóstico de enfermagem - Taxonomia NANDA}

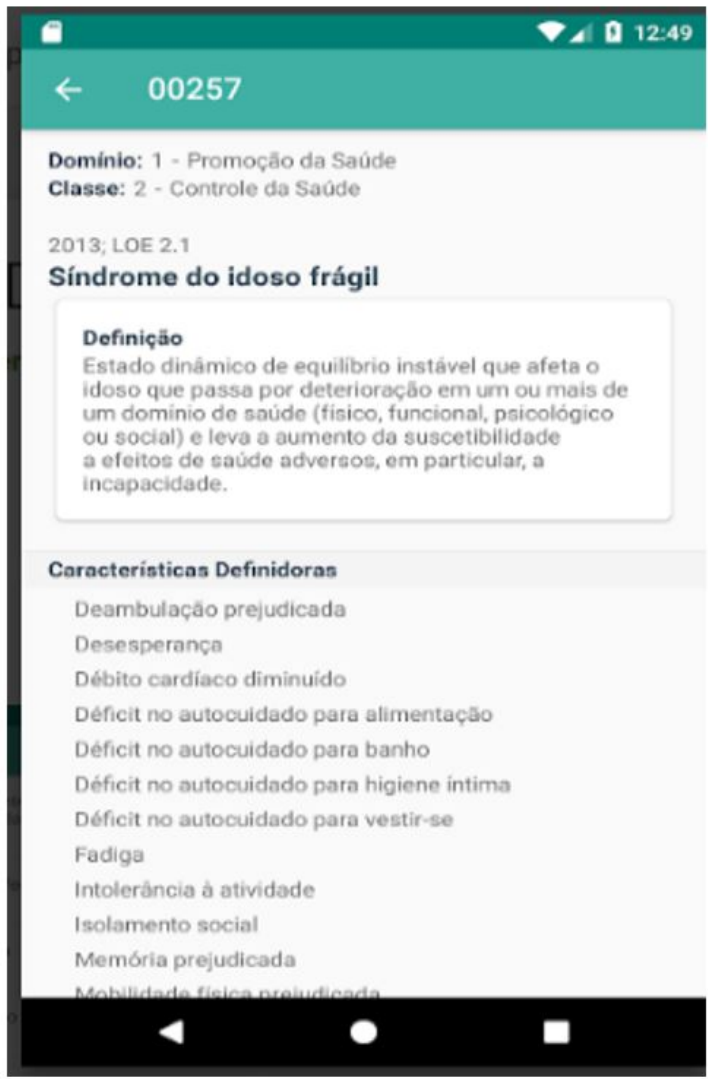

Fonte: Autoria Própria 
Figura 3 - Tela com as características definidoras

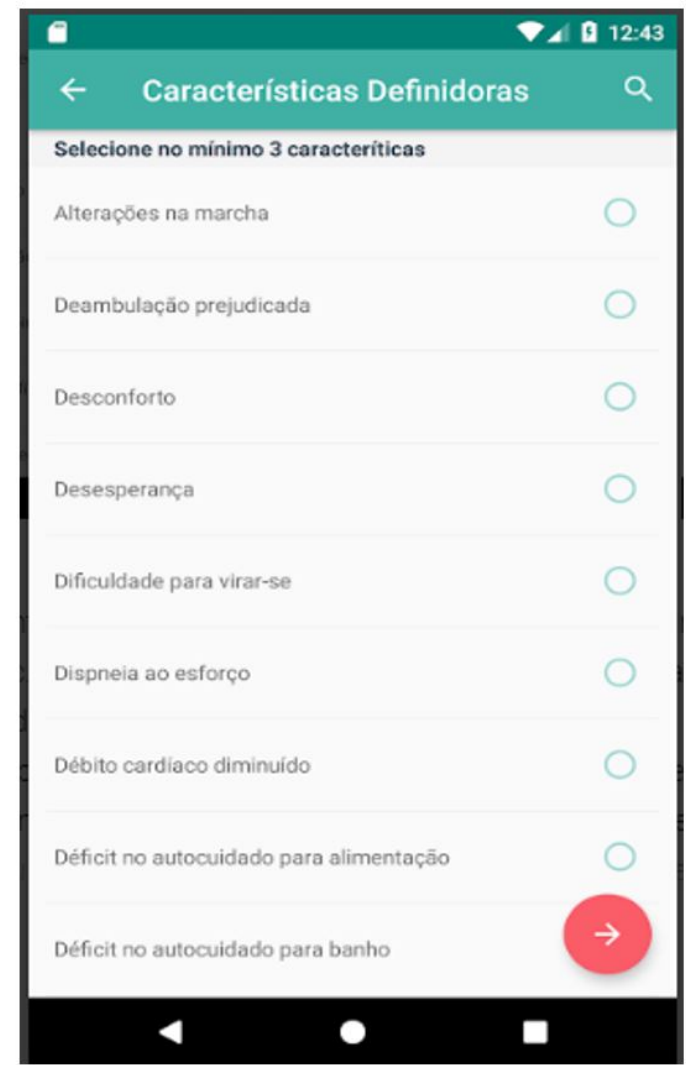

Fonte: Autoria Própria
Figura 4 - Resultado de Consulta pelas características definidoras

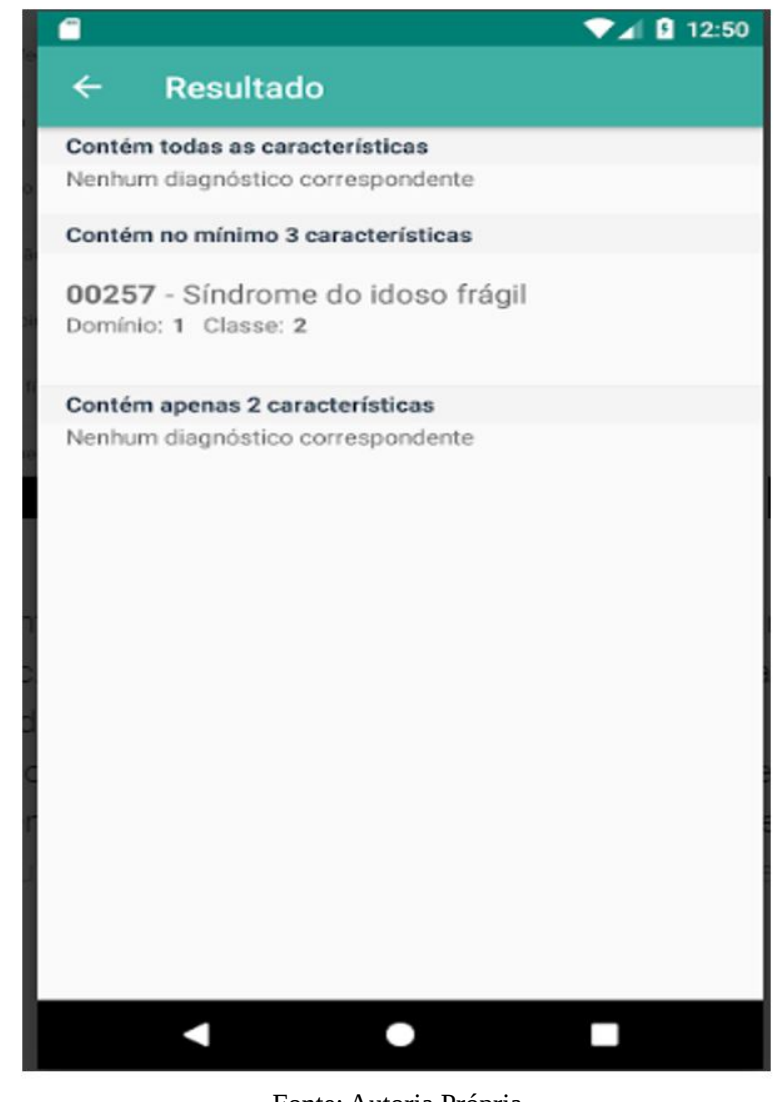

Fonte: Autoria Própria

Houve também uma avaliação sobre a percepção dos estudantes em relação ao uso do OA no processo ensino-aprendizagem da Taxonomia NANDA, essa avaliação se deu por meio de um instrumento de coleta de dados que contemplou as seguintes questões: Você teve alguma dificuldade em usar o objeto de aprendizagem? Depois de utilizar o objeto de aprendizagem você teve dificuldade de resolver o estudo de caso proposto? O objeto de aprendizagem pode contribuir para sua aprendizagem em relação ao diagnóstico de enfermagem? Como você avalia o conteúdo do objeto de aprendizagem? Você acha importante o uso da tecnologia da informação e comunicação na área da enfermagem? Como você avalia a interação com o OA (acessar as informações)? Havia ainda um espaço aberto para comentários e sugestões.

A análise quantitativa dos dados ocorreu com por meio de estatística descritiva com o uso do software Excel 2016 da empresa Microsoft Corporation. Para demonstrar que o uso do OA contribuiu significativamente no processo ensino-aprendizagem foi utilizado o teste de hipótese t Student, e para análise dos dados qualitativos utilizou-se a análise de conteúdo de Bardin.

\section{RESULTADOS E DISCUSSÃO}

Considerando as questões que foram delineadas para avaliar o perfil (dados demográficos) dos alunos, ressalta-se que no $5^{\circ}$ período há 19 (86,37\%) mulheres e 03 (13,63\%) homens, totalizando 22 alunos; no $7^{\circ}$ período tem-se $12(85,71 \%)$ mulheres e $02(14,29 \%)$ homens, totalizando 14 alunos e no $10^{\circ}$ período há 05 (83,33\%) mulheres e $01(16,67 \%)$ homem, totalizando 06 alunos. 
O resultado é semelhante há outros estudos presentes na literatura, como de Costa e Luz (2015) que encontrou predomínio do sexo feminino como perfil de alunos e tutores pesquisados, representando $68 \%$ dos alunos e $63,2 \%$ dos tutores.

Corroborando com os achados desse estudo, Freire (2016) evidencia que há inscritos nos Conselhos Regionais de Enfermagem (Coren) aproximadamente 1,8 mil profissionais de enfermagem (enfermeiros, técnicos e auxiliares), sendo que $85 \%$ desses profissionais são do sexo feminino, ainda que se verifique um progressivo aumento do número de homens.

Silva e Fernandes (2018) relatam que a predominância feminina é histórica e social na área da saúde e, especificamente, na Enfermagem que cuida da saúde das pessoas. Martins et al. (2006) inferem que esse predomínio do sexo feminino denota o traço cultural feminino como uma característica histórica e social da enfermagem que desde os primórdios as mulheres prestavam suas atividades na assistência hospitalar vinculada à higienização, considerada como extensão das atividades que possuíam como mulheres e, ainda prevalece o traço cultural feminino referente a força de trabalho que envolve o cuidado ao ser humano.

Na Tabela 1 apresenta-se a caracterização dos alunos considerando a idade, observa-se a predominância da faixa etária de 19 a 25 anos de idade, fato semelhante encontrado na literatura, pois de acordo com Corrêa et al. (2011) o ingresso no ensino superior se dá frequentemente entre 18 e 24 anos.

Tabela 1 - Idade dos alunos entrevistados classificados por faixa etária

\begin{tabular}{lccc}
\hline Faixa etária & $\mathbf{5}^{\mathbf{0}}$ período & $\mathbf{7}^{\mathbf{0}}$ período & $\mathbf{1 0}^{\mathbf{0}}$ período \\
\hline $19 \mid--25$ anos & 14 & 7 & 4 \\
\hline $25 \mid---30$ anos & 4 & 3 & 0 \\
\hline $30 \mid---35$ anos & 1 & 3 & 0 \\
\hline $35 \mid---40$ anos & 0 & 1 & 0 \\
\hline $40 \mid---45$ anos & 2 & 0 & 2 \\
\hline $45 \mid--50$ anos & 1 & 0 & 0 \\
\hline Total & $\mathbf{2 2}$ & $\mathbf{1 4}$ & $\mathbf{6}$ \\
\hline
\end{tabular}

Fonte: Autoria Própria

Na resolução do estudo de caso 1 observou-se que a nota média dos graduandos de enfermagem no pré-OA, ou seja, com conhecimento prévio e sem o uso do OA foi de 3,79 $\pm 2,14$, sendo que 03 (três) graduandos tiveram nota zero e 06 (seis) atingiram nota sete (maior nota obtida pelos alunos). No pós-OA a média foi 5,21 $\pm 2,74$, sendo que 10 (dez) graduandos tiveram nota oito (valor máximo obtido pelos alunos).

Quanto ao estudo de caso 2 observou-se que a nota média dos graduandos de enfermagem no pré-OA foi de 1,60 $\pm 1,19$, sendo que 10 (dez) graduandos tiveram nota zero no pré-OA, e quatro foi a maior nota obtida por somente dois graduandos. No pós-OA a média foi $2,79 \pm 1,44$, e nesta etapa 20 (vinte) graduandos alcançaram nota quatro (maior valor obtido pelos alunos).

Para validar estaticamente os resultados obtidos foi realizada a curva da distribuição normal para demonstrar que os resultados seguem um padrão de normalidade, formando uma curva Gaussiana, assim, foi possível avaliar os resultados por meio de um teste paramétrico, o teste t de Student.

Os resultados permitem afirmar que a maioria dos alunos aprendeu a utilizar o OA no processo ensino-aprendizagem da Taxonomia NANDA e obtiveram melhora nas avaliações realizadas após o uso do OA. 
Os resultados também demonstraram que antes de utilizar o OA, havia uma situação bimodal de acertos dos alunos (notas 4 e 5) no primeiro estudo de caso e após o uso do OA, a moda passou a ser 8, com 10 ocorrências; ressaltando-se que antes do uso do OA os alunos não alcançaram nota 8 . Também foi realizado o teste $t$ de Student, considerando-se um $p$-value de 0,05 ( $\alpha=5 \%)$, o $p$-value do P observado foi de 0,003 (0,3\%), permitindo rejeitar a hipótese nula, pois o resultado indica que as médias das duas avaliações não são iguais estatisticamente, portanto, confirma a eficácia do OA para resolução do estudo de caso 1.

Em relação ao segundo estudo de caso, antes de utilizar o OA, a moda era a nota 2 (13 ocorrências) e após o seu uso, a moda da nota passou a ser 4 (20 ocorrências). Também foi realizado o teste t de Student, considerando-se um $p$-value de $0,05(\alpha=5 \%)$, o $p$-value do P observado foi de 0,000 (0,0\%), permitindo rejeitar a hipótese nula, pois o resultado indica que as médias das duas avaliações não são iguais estatisticamente, assim, comprova a eficácia do OA também no estudo de caso 2.

Corroborando com os achados Colodetti (2018) realizou em seu estudo a validação de um app por um grupo composto por dez enfermeiras, com idades entre 28 e 50 anos, com experiência no tratamento de pacientes com úlceras em pés diabéticos e no manuseio de smartphones. Quando calculada a média das respostas do grupo das enfermeiras, as pontuações variaram entre 4,2 a 4,9. Desta forma, o app foi considerado adequado em todos os quesitos avaliados. Uma segunda análise das avaliações foi executada por meio das respostas, de usuários diferentes, para a mesma pergunta. Essas médias variaram de 4 a 5 .

No estudo de Domingues et al. (2015), os autores relatam que a maioria dos alunos aprendeu a utilizar o OA no processo ensino-aprendizagem e obtiveram melhora no aprendizado da Taxonomia NANDA, sendo que considerando uma amostra de 29 alunos participantes da simulação, 25 alunos $(86,2 \%)$ acertaram todos os itens e a média de acertos na atividade foi de 95,6\%.

No estudo analisado que versa sobre validação de um OA como protótipo digital de jogos educativos, Domingues et al. (2015) evidenciaram que $100 \%$ dos alunos afirmaram que os OA podem ser utilizados como um novo recurso de aprendizagem para o ensino profissionalizante de enfermagem, bem como pode-se incorporar jogos educativos em diversos temas da área da enfermagem visando um aprendizado ativo, participativo e estimulante para apreender os conteúdos ministrados no curso de graduação em enfermagem.

Santos et al. (2017) mencionam que a simulação em ambiente virtual não tem a pretensão de substituir as formas tradicionais de ensino utilizadas na saúde, porém apresenta-se como ferramenta auxiliar, com propostas e possibilidades que dependem da criatividade e do empenho de utilizadores e quando aplicadas em forma de jogos sérios, disponibilizam OA que podem garantir interação enriquecedora nos aspectos cognitivo e motor para os universitários, geração para quem essas tecnologias são muito mais atrativas e passíveis de motivar a aprendizagem.

Araújo et al. (2018) mencionam que o uso da TIC na área da saúde tem se mostrado uma estratégia inovadora, pois a versatilidade do seu emprego permite sua aplicabilidade para variados fins, favorecendo a aproximação entre o sujeito cuidado e o profissional cuidador.

Na sequência são apresentados os resultados referentes às questões que permitiram a avaliação do uso do OA, bem como sua importância no processo ensino-aprendizagem, de acordo com os participantes da pesquisa.

Na questão "Você teve alguma dificuldade em usar o objeto de aprendizagem?”, 01 (2,38\%) assinalou dificuldade extrema, 02 (4,76\%) responderam muita dificuldade, 10 (23,8\%) dificuldade moderada, 20 (47,63\%) pouca dificuldade e 09 (21,43\%) relataram que não tiveram dificuldades. Portanto, 29 (69,06\%) participantes assinalaram que não encontraram ou foram poucas as dificuldades para usar o AO. 
Resultado semelhante foi encontrado no estudo de Carneiro e Silveira (2012), uma vez, que 89,5\% dos participantes consideraram muito fácil ou fácil o uso do OA apresentado para eles.

Costa e Luz (2015) relatam que visando garantir a atenção do aluno em relação ao conteúdo apresentado, deve-se evitar sobrecarga de informações disponíveis nas telas e apresentar apenas o que é relevante para o aluno naquele momento buscando sequenciar de forma lógica as informações, utilizando uma linguagem na interface com simplicidade, clareza e objetividade, tornando os conteúdos mais acessíveis ao usuário e motivacional para os alunos que possuem dificuldades para usar OA.

Em relação à pergunta "Depois de utilizar o objeto de aprendizagem você teve dificuldade de resolver o estudo de caso proposto?”, 01 (2,38\%) relatou dificuldade extrema, 02 (4,76\%) relataram muita dificuldade, 09 (21,43\%) dificuldade moderada, 19 (45,24\%) pouca dificuldade e $11(26,19 \%)$ responderam que não tiveram dificuldade para resolver os estudos de caso propostos. Observa-se que $30(71,43 \%)$ registraram que tiveram pouca ou nenhuma dificuldade para resolver as atividades avaliativas propostas, após utilizar o OA.

Carneiro e Silveira (2012) realizaram estudo sobre a aprendizagem com OA, 54\% indicaram que o OA ajudou e $38 \%$ apontaram que o OA ajudou muito a compreender o assunto, totalizando $92 \%$ dos participantes com respostas afirmativas.

Domingues et al. (2015) relatam que a enfermagem tem a necessidade de inovações que estimulem o conhecimento e a aprendizagem autônoma e independente no processo ensino-aprendizagem, por meio da adoção de OA, pois evidencia-se que o uso de TIC permite a realização de processos de ensinagem mais criativos, oportunizando a aprendizagem mais ativa por parte dos alunos, vencendo as dificuldades de aprendizagem com apoio tecnológico.

Na questão "O Objeto de Aprendizagem pode contribuir para sua aprendizagem em relação ao diagnóstico de enfermagem?”, 01 (2,38\%) discordou quanto a contribuição, 01 (2,38\%) relatou não discordar, mas não concordar com o aprendizado, 18 (42,86\%) concordaram quanto a contribuição e 22 (52,38\%) concordaram totalmente com a contribuição do OA no processo ensino-aprendizagem em relação ao diagnóstico de enfermagem. Em síntese, 40 (95,24\%) dos participantes consideraram que o OA foi importante para o processo ensino-aprendizagem relativo ao diagnóstico de enfermagem.

Quando perguntados “Como você avalia o conteúdo do objeto de aprendizagem? ”, 01 (2,38\%) relatou ruim, 08 (19,04\%) avaliaram como satisfatório, 8 (19,04\%) bom e 25 (59,54\%) avaliaram muito bom, assim, pode-se enfatizar que $41(97,62 \%)$ dos participantes gostaram do conteúdo do OA.

Peres, Meira e Leite (2007) descreveram a opinião dos alunos sobre a avaliação do conteúdo do OA e apresentou como resultado que a avaliação dos estudantes quanto à forma, o conteúdo e a utilização do site como ambiente de ensino foram de bom a muito bom, pelo fato de ampliar e diversificar as formas de comunicação entre discentes e docentes, permitindo a aquisição de novos conteúdos, além de facilitar o aprendizado.

Blasca et al. (2013) ressaltam que 71,5\% dos participantes avaliaram a compreensão do conteúdo como "excelente". Este dado demonstra que o OA do estudo favorece a aquisição do conhecimento, tornando-se uma ferramenta valiosa no processo ensino-aprendizagem. Em relação à presença de imagens e vídeos que esclareçam o conteúdo, 21,4\% dos participantes avaliaram como "excelente" e a opinião dos alunos foi positiva, demonstrando índice de alta aprovação, trazendo aos alunos a construção do seu próprio conhecimento e desenvolvendo um pensamento crítico e reflexivo.

Na questão "Você acha importante o uso da tecnologia da informação e comunicação na área da enfermagem?”, 01 (2,38\%) não considerou importante, 10 (23,82\%) consideraram importante e 31 (73,80\%) concorda- 
ram totalmente sobre a importância do uso da tecnologia da informação na área da enfermagem. Portanto, 41 (97,62\%) acreditaram que o uso da TIC na área de enfermagem é importante ou ainda imprescindível.

No estudo de Rezende, Santos e Medeiros (2016) as alunas mostraram que há consenso entre as enfermeiras quanto à importância de um aplicativo para a SAE em tecnologia móvel, principalmente, no que se refere a mobilidade e agilidade para realizar a evolução do paciente e elaborar o plano de cuidados, otimizando, assim, o tempo e flexibilizando a gerência das ações de cuidado com o uso de tablet.

Em relação à pergunta "Como você avalia a interação com o objeto de aprendizagem (acessar as informações)?”, 09 (21,43\%) assinalaram como satisfatória, 14 (33,33\%) relataram boa e 19 (45,24\%) como muito boa. Conforme pode-se observar, $100 \%$ dos participantes, avaliaram positivamente a interação com o OA; fato este também encontrado por Silveira et al. (2010), uma vez que na avaliação dos alunos que utilizaram um OA no estudo desses autores, observou-se, pelos resultados, que os OA, inseridos no Moodle satisfizeram os usuários, foram adequados, levaram a resultados precisos ou dentro do esperado e ainda aumentaram e estimularam o aprendizado dos alunos.

Silva, Taleb e Costa (2015) enfatizaram que o processo de navegação no ambiente virtual orienta caminhos propondo atividades e disponibiliza múltiplas experiências, criando condições para que os pesquisados busquem e experimentem diferentes situações de troca e compartilhamento de conhecimentos, tendo por base os casos clínicos apresentados, permitindo um material de apoio que possibilita a reflexão. Os ambientes virtuais desenvolvem espaços de construção e colaboração interativos, consolidando as informações para que o graduando consiga apreender o conteúdo de forma dinâmica, formativa e interativa.

Considerando a questão “Como você avalia a sua familiaridade como o objeto de aprendizagem?”, 04 (9,53\%) responderam ruim, 09 (21,43\%) responderam como satisfatória, 21 (50\%) avaliaram como boa e 08 $(19,04 \%)$ avaliaram como muito boa, totalizando, assim 38 (90,47\%) respostas favoráveis em relação a familiaridade na utilização do OA.

Domingues et al. (2015) mencionam que os recursos tecnológicos aliados à educação na área da saúde têm se mostrado bastante eficientes, e é possível observar ainda que as gerações atuais, como a Geração Z, se encontram muito mais imersas e possuem maior facilidade em lidar com as tecnologias, demonstrando preferência pelo uso de recursos tecnológicos quando comparados às estratégias tradicionais de ensino-aprendizagem.

Na última questão que permitia a avaliação qualitativa, disponibilizada para sugestões e comentários, foram encontradas três categorias que são apresentadas no Quadro 1.

\section{Quadro 1 - Contribuições e comentários dos entrevistados da amostra referente ao uso de OA no processo ensino-aprendizagem do diagnóstico de enfermagem.}

\begin{tabular}{|l|l|}
\hline Contribuições/satisfações & Total \\
\hline Inserir mais diagnósticos de enfermagem & 04 \\
\hline Contribui muito para nosso aprendizado & 05 \\
\hline Inovação, praticidade, agilidade nos serviços de enfermagem & 01 \\
\hline
\end{tabular}

Fonte: Autoria Própria

Constata-se que são relevantes as citações dos participantes demonstrando a motivação e interesse, bem como a inserção de todos os diagnósticos de enfermagem para uso na assistência de enfermagem, cabe destacar que os achados desse estudo são semelhantes aos apresentados por Fonseca et al. (2015), sendo que os pesquisados desejaram novos desafios e inserção de outros temas da enfermagem no OA, o que reflete em motivação para o estudo e interesse dos estudantes pelo uso da ferramenta digital. 
As contribuições descritas pelos graduandos em enfermagem no estudo de Martins et al. (2016), versam sobre a rápida evolução de novas tecnologias que promovam mudanças na forma de acessar e organizar informações e trazem um relevante avanço na construção do conhecimento, tornando-se significativa ferramenta pedagógica. As TIC são utilizadas no ensino de enfermagem e no gerenciamento da assistência e têm modificado as concepções de ensino-aprendizagem trazendo novas possibilidades de fontes de pesquisa, permitindo um ensino mais eficaz e atualizado.

A avaliação positiva dos graduandos em relação à aceitação, inovação e praticidade com o OA reforçam as vantagens de motivação para o aprendizado que as tecnologias educacionais digitais podem oferecer.

A agilidade nos serviços de enfermagem com o uso do OA, citada pelos graduandos de enfermagem, demonstra que estão assumindo uma postura de abertura ao novo, centrados nas necessidades específicas dos futuros profissionais, em que a tecnologia com abordagens educacionais diversificadas propiciam uma alternativa que aponta para um novo caminho no que diz respeito à formação de profissionais de enfermagem, ressaltando a integração das TIC no cotidiano dos serviços de enfermagem, trazendo a evolução no desenvolvimento das atividades inerentes a todos desta equipe, aprimorando a excelência nos atendimentos ao paciente, família e coletividade (PACHECO; AZAMBUJA; BONAMIGO, 2017).

\section{CONCLUSÃO}

Os resultados demonstram que o OA desenvolvido e validado é uma ferramenta útil para ser utilizada no processo ensino-aprendizagem na definição do diagnóstico de enfermagem através da Taxonomia NANDA, pois considerando as notas dos pós-OA dos estudos de caso 1 e 2 pode-se constatar que o conhecimento dos graduandos de enfermagem que participaram do estudo melhorou, pois as notas aumentaram após o uso do OA elaborado.

A maioria dos alunos concordou que a utilização do OA facilitou o processo ensino-aprendizagem relativo a Taxonomia NANDA, indicando, assim, que adotar OA como recurso didático-pedagógico nos cursos de graduação em Enfermagem corrobora para a motivação dos alunos e apoia o desenvolvimento cognitivo para a competência referente à realização da assistência de enfermagem.

Conclui-se que o OA desenvolvido e disponibilizado na Google Play Store é uma ferramenta que tem aplicação no ensino do diagnóstico de enfermagem pela Taxonomia NANDA e pode contribuir para a formação de profissionais de enfermagem, em diferentes modalidades de ensino, podendo auxiliar na formação de profissionais de enfermagem.

\section{REFERÊNCIAS}

ARAÚJO, F. A. C. et al. Elaboração de Software para Tomada de Decisões Clínicas em Enfermagem na Prevenção de Quedas em Pediatria. Atas do $7^{\circ}$ Congresso Ibero-Americano em Investigação Qualitativa CIAIQ2018, v. 2, p.1276- 1281, 2018.

ALVAREZ, A. G.; DAL SASSO, G. T. M. Objetos virtuais de aprendizagem: contribuições para o processo de aprendizagem em saúde e enfermagem. Acta Paulista de Enfermagem. São Paulo, v. 24, n. 5, p. 707-11, 2011.

BARROS, A. L. B. L.; FAKIH, F. T.; MICHEL, J. L. M. O uso do computador como ferramenta para a implementação do processo de enfermagem: a experiência do Hospital São Paulo/UNIFESP. Revista Brasileira de Enfermagem. Brasília, v. 55, n. 6, p.714-719, nov.dez.2002. 
BLASCA, W. Q. et al. Projeto Jovem Doutor Bauru: capacitação de estudantes do ensino médio em saúde auditiva. Revista CEFAC. Campinas, v. 15, n. 6, p. 1407-1417, 2013.

CARNEIRO, M. L. F.; SILVEIRA, M. S. Objetos de aprendizagem sob o ponto de vista dos alunos: um estudo de caso. Revista Novas Tecnologias na Educação - RENOTE. Porto Alegre, v. 10, n. 3, p. 1-9, 2012.

CLEIRES, A. B. B. et al. Análise do conteúdo de uma tecnologia para raciocínio diagnóstico de enfermagem. Revista Brasileira de Enfermagem. Brasília, v. 68, n. 2, p. 261-268, mar-abr 2015.

COLODETTI, R. Cuidado Tópico da Úlcera do Pé Diabético: aplicativo móvel para subsídio à tomada de decisão. 2018. 140 p. Dissertação (Mestrado Profissional em Enfermagem). Centro de Ciências da Saúde. Universidade Federal do Espírito Santo, Vitória/ES, 2018.

CONSELHO FEDERAL DE ENFERMAGEM - COFEn. Resolução COFEn $n^{\circ}$ 358/2009. Dispõe sobre a Sistematização da Assistência de Enfermagem e a implementação do Processo de Enfermagem em ambientes, públicos ou privados, em que ocorre o cuidado profissional de Enfermagem, e dá outras providências. Brasília (Brasil): Conselho Federal de Enfermagem, 2009.

CORRÊA, A. K. et al. Perfil de estudantes ingressantes em licenciatura: Escola de Enfermagem de Ribeirão Preto da Universidade de São Paulo. Revista da Escola de Enfermagem da USP. São Paulo, v. 45, n. 4, p. 933-938, 2011.

COSTA, C. P. V.; LUZ, M. H. B. A. Objeto virtual de aprendizagem sobre o raciocínio diagnóstico em enfermagem aplicado ao sistema tegumentar. Revista Gaúcha de Enfermagem. Porto Alegre, v. 36, n. 4, p. 55-62, 2015.

DAL SASSO, G. T. M. et al. Processo de enfermagem informatizado: metodologia para associação da avaliação clínica, diagnósticos, intervenções e resultados. Revista da Escola de Enfermagem da USP. São Paulo, v. 47, n. 1, p. 242-249, 2013.

DOMINGUES, A. N. et al. Jogo educacional sobre segurança do paciente: avaliação de estudantes de enfermagem. In: XX Congresso Internacional de Informática Educativa (TISE 2015), p. 684-688, 2015.

FREIRE, N. P. Trajetória: fatos e fotos da pesquisa perfil da enfermagem. Enfermagem em Foco. Brasília, v. 7, n. esp., p. 81-88, 2016.

FONSECA, L. M. M. et al. Impact of the Use of a Digital Learning Object in the Teaching of Clinical Assessment of Preterm Infants: A Comparative. Procedia - Social and Behavioral Sciences. v. 46, p. 1192-1197, 2012.

FONSECA, L. M. M. et al. Serious game e-Baby: percepção dos estudantes de enfermagem sobre a aprendizagem da avaliação clínica do bebê prematuro. Revista Brasileira de Enfermagem. Brasília, v. 68, n. 1, p. 13-19, 2015.

HORTA, V. A. Processo de Enfermagem. São Paulo: EPU, 1979.

MARTINS, C. et al. Perfil do enfermeiro e necessidades de desenvolvimento de competência profissional. Enseñanza. Florianópolis, v. 15, n. 3, p. 472-478, 2006.

MARTINS, N. P. et al. Úlcera por pressão: avaliação de software para elaboração de conteúdo interativo para graduandos em enfermagem. Revista Saúde-UNG-Ser. Guarulhos, v. 9, n. 1-2, p. 43-53, 2016.

MILLÃO, L. F. et al. Integração de tecnologias digitais no ensino de enfermagem: criação de um caso clínico sobre úlceras por pressão com o software SIACC. RECIIS - Revista Eletrônica de Comunicação, Informação e Inovação em Saúde. Rio de Janeiro, v. 11, n. 1, p. 1-12, jan.-mar. 2017. 
PACHECO, K. C. F.; AZAMBUJA, M. S.; BONAMIGO, A. W. A construção de objeto de aprendizagem sobre doenças transmissíveis para agentes comunitários de saúde. Revista Gaúcha de Enfermagem. Porto Alegre, v. 38, n. 4, p. 1-9. 2017.

PAGANIN, A. et al. Implantação do diagnóstico de enfermagem em unidade de terapia intensiva: uma análise periódica. Revista Gaúcha de Enfermagem. Porto Alegre, v. 31, n. 2, p. 307-313, 2010.

PERES, H. H. C.; MEIRA, K. C.; LEITE, M. M. J. Ensino de didática em enfermagem mediado pelo computador: avaliação discente. Revista da Escola de Enfermagem da USP. São Paulo, v. 41, n. 2, p. 271-278, 2007.

REZENDE, L. C. M.; DOS SANTOS, S. R.; MEDEIROS, A. L. Avaliação de um protótipo para Sistematização da Assistência de Enfermagem em dispositivo móvel. Revista Latino-Americana de Enfermagem. Ribeirão Preto, v. 24, e2714, 2016.

SABOIA, J.; VARGAS, P. L.; VIVA, M. A. A. O uso dos dispositivos móveis no processo de ensino e aprendizagem no meio virtual. Revista Cesuca Virtual: conhecimento sem fronteiras. Rio Grande do Sul, v. 1, n. 1, p. 1-13, 2013.

SALVADOR, P. T. C. O. et al. Objeto e ambiente virtual de aprendizagem: análise de conceito. Revista Brasileira de Enfermagem. Brasília, v. 70, n. 3, p. 599-606, mai-jun 2017.

SAMPAIO, R. L.; ALMEIDA, A. R. S. Aprendendo matemática com objetos de aprendizagem. Ciências \& Cognição. Rio de Janeiro, v. 15, n. 1, p. 64-75, 2010.

SANTOS, C. A. et al. Jogos sérios em ambiente virtual para ensino-aprendizagem na saúde. Northeast Network Nursing Journal. Fortaleza-CE, v. 18, n. 5, p. 702-709, 2017.

SILVA, F. B. M.; FERNANDES, M. I. M. Perfil Epidemiológico dos Acidentes com Material Biológico no Município de Maringá/Paraná-2014. Revista Uningá Review. Maringá-PR, v. 29, n. 3, p. 52-59, 2018.

SILVA, E. C.; TALEB, A. C.; COSTA, N. M. S. C. Ambiente virtual de avaliação de competências no manejo do diabetes mellitus. Revista Brasileira de Educação Médica. Brasília, v. 39, n. 3, p. 470-478, 2015.

TUBELO, R. A. et al. The Influence of a Learning Object with Virtual Simulation for Dentistry: a randomized controlled trial. International Journal of Medical Informatics. v. 85, n. 1, p. 68-75, 2012.

WESTIN, U. et al. Sistematização da Assistência de Enfermagem: uma proposta de webquest. SIED: EnPED - Simpósio Internacional de Educação a Distância e Encontro de Pesquisadores em Educação a Distância. São Carlos, 2016. 